\title{
OptSortSph: Optical Sorting in a Standing Wave Field Calculated with Effective Velocities and Diffusion Constants
}

\author{
Zachary H. Levine and John J. Curry \\ National Institute of Standards and Technology, \\ Gaithersburg, MD 20899 \\ zlevine@nist.gov \\ john.curry@nist.gov
}

Software Download: http://doi.org/10.18434/T4SW26

Software Version: 1.0 .

Key words: dielectric spheres; gradient force; Mie scattering; optical sorting; standing-wave laser field.

Accepted: August 30, 2016

Published: September 7, 2016

http://dx.doi.org/10.6028/jres.121.020

\section{Summary}

Recently, as part of a study of the ability of a Gaussian standing-wave interference field to sort small dielectric spheres entrained in a moving fluid, we implemented a two-length-scale method to determine the trajectories of the particles. In the first step, effective velocities and diffusion constants are determined by solving the Fokker-Planck equation in a force field determined by the Maxwell stress tensor. In the second step, these effective constants of motion are used to predict positions of particles swept across the interference field. Here, we present the code which was used in a companion article [1].

The fine scale tracks a domain of typically 16 interference fringes with a spatial resolution typically 1/16 of a fringe. The Fokker-Planck equation tracks the evolution of the probability distribution of a sphere in a period set of one-dimensional sinusoidal wells. Physically, these sinusoidal wells exist because of the interaction between the dielectric particle and the electromagnetic standing wave. The effective velocity is determined by finding the mean position of the sphere as a function of time. After allotting time for intrawell equilibration, the mean position becomes a linear function of time. The slope of this line is the velocity. Similarly, the diffusion constant is related to the time evolution of the second moment of the distribution. The effective velocity and diffusion constant needs to be found for a sphere of a given radius for a range of optical intensities.

The coarse scale is given by the size of the width of the standing wave, typically $3 \mathrm{~mm}$. Although the position is a continuous variable, the solution is unaware of the fringes except to the extent that there is a position-dependent effective velocity and effective diffusion constant which is determined on the fine scale above. The crossing time is a simple function of the transverse distance between the starting position and end line. A simple approximation to the distribution of crossing times (induced by diffusion) is made, however, uniform motion across the interference field is assumed. The distance travelled longitudinally is found by integrating the effective velocity across the Gaussian envelope of the interference field. The 
diffusion constant is integrated as well to find the standard deviation of the position at the exit. A correction is made for the standard deviation of the crossing time.

By default, the notebook is configured to reproduce some of the more interesting results of Ref. [1], specifically the particle radii for which the position on exit is a very sensitive function of the radius. The user is encouraged to explore other configurations.

\section{Software Specifications}

\begin{tabular}{|l|l|}
\hline NIST Operating Unit(s) & Sensor Science Division, Physical Measurement Laboratory \\
\hline Category & Fokker-Planck equation with extraction and use of effective velocity and diffusion constants \\
\hline Targeted Users & Researchers interested in designing continuous optical sorting systems \\
\hline Operating System(s) & Cross-platform, where Mathematica is installed \\
\hline Programming Language & Mathematica 10.0 \\
\hline Inputs/Outputs & I/O is described in the Mathematica notebook \\
\hline Documentation & The Mathematica notebook is self-documenting \\
\hline Accessibility & N/A small-scale research tool \\
\hline Disclaimer & https://www.nist.gov/disclaimer \\
\hline
\end{tabular}

\section{Methods}

There are two classes of tests for this software: extrinsic and intrinsic. The extrinsic test is a comparison to a Monte Carlo program which is not supplied, but is described in Ref. [1]. Substantial agreement has been obtained. The intrinsic tests are described in the Appendix of Ref. [1]. The most difficult part of the code is the solution of the Fokker-Planck equation. There are three parameters. If any one of the three is set to zero, there is an analytic solution. The code has been compared to such analytic solutions in all cases.

\section{References}

[1] Curry JJ and Levine ZH (2016) Continuous feed optical sorting of aerosol particles. Optics Express 24:14100-14123. http://dx.doi.org/10.1364/OE.24.014100

About the authors: Zachary Levine is a Physicist at NIST and a Fellow of the American Physical Society. His work has concentrated on the interaction of light and other probes with matter. John Curry is an Electrical Engineer at NIST. His current interests include manipulation and measurement of particles using optical forces. The National Institute of Standards and Technology is an agency of the U.S. Department of Commerce. 